\title{
Staging performance of whole-body DWI, PET/CT and PET/MRI in invasive ductal carcinoma of the breast
}

\author{
ONOFRIO ANTONIO CATALANO ${ }^{1,2}$, DANIA DAYE ${ }^{1}$, ALBERTO SIGNORE $^{3,11}$, CARLO IANNACE $^{4}$, \\ MARK VANGEL $^{2}$, ANGELO LUONGO $^{5}$, MARCO CATALANO $^{6}$, MAZZEO FILOMENA $^{7}$, \\ LUIGI MANSI ${ }^{8}$, ANDREA SORICELLI $^{9}$, MARCO SALVATORE $^{10}$, NICCOLO FUIN $^{2}$, \\ CIPRIAN CATANA $^{2}$, UMAR MAHMOOD ${ }^{1,2}$ and BRUCE ROBERT ROSEN ${ }^{2}$
}

\begin{abstract}
${ }^{1}$ Department of Radiology, Massachusetts General Hospital, Harvard Medical School, Boston, MA 02114; ${ }^{2}$ Martinos Center for Biomedical Imaging, Massachusetts General Hospital, Harvard Medical School, Charlestown, MA 02129, USA; ${ }^{3}$ Department of Nuclear Medicine, University of Roma 'La Sapienza', Rome, RM 00161; ${ }^{4}$ Breast Unit, Ospedale Moscati, Contrada Amoretta, Avellino, AV 83010; ${ }^{5}$ Department of Radiology, Gammacord, Benevento, BN 82100; ${ }^{6}$ Department of Radiology, University of Naples 'Federico II', Napoli, NA 80131; ${ }^{7}$ Department of Biology and Pathology, University of Naples 'Parthenope', Naples, NA 80131; ${ }^{8}$ Department of Nuclear Medicine, Second University of Naples, Napoli, NA 80130; 'Department of Diagnostic Imaging, University of Naples 'Parthenope', Napoli, NA 80131; ${ }^{10}$ Diagnostic Imaging, SDN, Napoli, NA 80131, Italy; ${ }^{11}$ Department of Nuclear Medicine and Molecular Imaging, University Medical Center Groningen, University of Groningen, Groningen, The Netherlands
\end{abstract}

Received March 20, 2017; Accepted May 11, 2017

DOI: $10.3892 /$ ijo.2017.4012

\begin{abstract}
The aim of the present study was to evaluate the performance of whole-body diffusion-weighted imaging (WB-DWI), whole-body positron emission tomography with computed tomography (WB-PET/CT), and whole-body positron emission tomography with magnetic resonance imaging (WB-PET/MRI) in staging patients with untreated invasive ductal carcinoma of the breast. Fifty-one women with newly diagnosed invasive ductal carcinoma of the breast underwent WB-DWI, WB-PET/CT and WB-PET/MRI before treatment. $A$ radiologist and a nuclear medicine physician reviewed in consensus the images from the three modalities and searched for occurrence, number and location of metastases. Final staging, according to each technique, was compared. Pathology and imaging follow-up were used as the reference. WB-DWI, WB-PET/CT and WB-PET/MRI correctly and concordantly staged 33/51 patients: stage IIA in 7 patients, stage IIB in 8 patients, stage IIIC in 4 patients and stage IV in 14 patients. WB-DWI, WB-PET/CT and WB-PET/MRI incorrectly and concordantly staged $1 / 51$ patient as stage IV instead of IIIA. Discordant staging was reported in 17/51 patients. WB-PET/MRI resulted in improved staging when compared to WB-PET/CT (50 correctly staged on WB-PET/MRI
\end{abstract}

Correspondence to: Professor Onofrio Antonio Catalano, Department of Radiology, Massachusetts General Hospital, Harvard Medical School, 55 Fruit Street, Boston, MA 02114, USA

E-mail: onofriocatalano@yahoo.it

Key words: PET/MR, breast cancer, PET/CT, staging, diffusionweighted imaging vs. 38 correctly staged on WB-PET/CT; McNemar's test; $\mathrm{P}<0.01)$. Comparing the performance of WB-PET/MRI and WB-DWI (43 correct) did not reveal a statistically significant difference (McNemar test, $\mathrm{P}=0.14$ ). WB-PET/MRI is more accurate in the initial staging of breast cancer than WB-DWI and WB-PET/CT, however, the discrepancies between WB-PET/MRI and WB-DWI were not statistically significant. When available, WB-PET/MRI should be considered for staging patient with invasive ductal breast carcinoma.

\section{Introduction}

Imaging plays a pivotal role in the staging and management of breast cancer patients. For instance, breast MRI is used in stage I disease to rule out additional sites of malignancy that might be occult at mammography. Other imaging techniques, such as bone scans, abdominal CT or MR and chest CT are recommended in stage I-IIB disease in patients with abnormal liver function tests, alkaline phosphatase, bone pain, abnormal physical examination, localized bone pain, or with abdominal or pulmonary symptoms and in stage IIIA disease. PET/CT is considered optional for stage IIIA, stage IV and recurrent disease (1).

Growing evidence suggests that PET/CT may detect distant metastases (sensitivity of 78-100\%) over conventional nonmetabolic imaging modalities (sensitivity of 37-78.6\%) (2). Specifically, a recent meta-analysis showed that the detection of distant metastases increases from 1.2 to $3.3-34.3 \%$ if $\mathrm{PET} / \mathrm{CT}$ is added to conventional imaging for the staging of patients with stage II breast cancer (2).

The use of diffusion-weighted imaging (DWI) MRI has recently increased as a potential alternative to PET/CT for whole-body staging, prognosis and treatment response assess- 
ment of several malignancies, including malignancies of the breast. Some studies suggest comparable performance of DWI to PET in disease staging with the added advantages of lack of radiation, widespread availability and no additional costs over those related to operation of a normal MR scanner $(3,4)$. Other studies have found similar sensitivity of DWI to metabolic imaging at the expense of reduced accuracy (4-8). Therefore in this study we also explored, as described below, the performance of DWI standing alone.

In recent years, PET/MRI has emerged as a new tool with significant clinical potential for the evaluation and management of cancer patients (9-12). PET/MRI allows for improved diagnosis and staging accuracy in a number of primary and metastatic cancers, including lymphoma, head and neck, liver and bone tumors (13-19). Moreover, data suggest that PET/MRI might play a superior role in affecting oncologic management decisions, compared to PET/CT $(20,21)$.

Available data supporting the use of PET/MRI in the evaluation of breast cancer patients remain limited but has been promising (22-25). While primary lesion detection has been previously shown to be equivalent between PET/CT and PET/MRI, PET/MRI might improve detection of metastatic lesions, when compared to PET/CT and might lead to management changes in up to one third of the patients, when compared to initial clinical staging $(23,26)$. Other studies have also shown a role of combining PET and MRI in characterizing tumor pathology and predicting response to therapy (27-30).

The aim of the present study was to compare the staging performance of whole body diffusion-weighted imaging (WB-DWI), whole body positron emission tomography with computed tomography (WB-PET/CT), and whole body positron emission tomography with magnetic resonance imaging (WB-PET/MRI) in staging breast cancer patients with newly diagnosed invasive ductal carcinoma. To the best of our knowledge, this is the first study concurrently assessing the performance of these three modalities in the same patient population.

\section{Materials and methods}

Study design and patient enrollment. A retrospective HIPAAcompliant study was approved by the institutional review board. Informed consent, that included the possibility of subsequent usage of imaging and clinical data for imaging research purposes, was obtained from patients before undergoing same day PET/MRI and PET/CT. Inclusion criteria consisted of: i) new, untreated biopsy-proven invasive ductal carcinoma of the breast; ii) female; iii) 18 years of age or older; iv) clinical contrast enhanced (CE) PET/CT study; v) same day CE-PET/MRI study; and vi) availability of pathology or at least two-years imaging follow-up. Patients were excluded if they met any of the following criteria: i) pregnancy; ii) blood glucose >140 mg/dl; iii) contraindication to MR imaging; and iv) inclusion in previous PET/MRI studies.

\section{Imaging protocols}

PET/CT imaging. PET/CT images were acquired $\sim 1 \mathrm{~h}$ following FDG administration, mean FDG activity of $4.44 \mathrm{MBq} / \mathrm{kg}$ of body weight. Whole body images were acquired using a 64-detector row PET/CT scanner (Gemini TF; Philips
Medical Systems, Best, The Netherlands) with time-of-flight capability. Automatic attenuation correction was performed using attenuation correction maps generated from CT imaging. Both non-contrast and contrast-enhanced CT images were collected. Iopamidol (Iopamiro 370; Bracco Imaging, Milan, Italy) was injected intravenously using a power injector at a rate of $2 \mathrm{ml} / \mathrm{sec}$ with a dose of $80 \mathrm{ml}$ in patients weighing $<80 \mathrm{~kg}$; and a dose of $100 \mathrm{ml}$ in patients weighing $>80 \mathrm{~kg}$. Bolus care function was used to acquire diagnostic quality arterial phase images of the upper abdomen, portal venous phase images of the whole body and delayed phase of the abdomen and pelvis.

PET/MRI imaging. PET/MRI images were acquired using a Biograph mMR imager (Siemens Healthcare, Erlangen, Germany) with a 16-channel head and neck surface coil and three or four 12-channel body coils, depending on the patient's height. The coils were combined to form a whole-body coil using total imaging matrix technology. PET/MRI images were collected $\sim 1.5 \mathrm{~h}$ following FDG injection. The following MRI sequences were obtained concurrently with PET: axial DWI (b-values 50, 400 and $800 \mathrm{~s} / \mathrm{mm}^{2}$ ), coronal short tau inversion recovery (STIR), coronal T1-weighted Dixon, axial T2 weighted half Fourier acquired single-shot turbo spin echo (HASTE). Contrast enhanced-axial and coronal T1-weighted fat saturated (VIBE, volume interpolated breath-hold examination) images were acquired after PET completion. PET attenuation correction was performed using the twopoint Dixon sequence. For contrast-enhanced MR imaging, $0.1 \mathrm{mmol} / \mathrm{kg}$ of gadopentetate dimeglumine (Magnevist; Bayer Schering Pharma, Berlin, Germany) was injected at a rate of $3 \mathrm{ml} / \mathrm{sec}$ followed by the same volume of saline at the same rate, using a power injector. The total time of PET/MRI imaging was $\sim 1 \mathrm{~h}$.

Image post-processing. PET/CT image post-processing was performed using a dedicated workstation (Extended Brilliance Workstation Philips). Post-processing of PET/MRI images was done using a Syngovia workstation (Siemens Healthcare). Image post-processing consisted of image co-registration and fusion. Images were archived using the IDS7 image archiving and communication system (Sectra, Linkoping, Sweden).

Image interpretation. WB-PET/CT, WB-DWI standing alone, WB-PET/MRI that also included DWI and ADC maps, were randomly presented and evaluated separately, at least 6 weeks apart, in consensus by a radiologist (OAC) with 17 years of experience in MR and 5 years in nuclear medicine and a nuclear medicine physician (AS) with 33 years of experience in nuclear medicine and 20 years in MR. Specifically, they searched for the occurrence, number and location of metastatic lesions and recorded for each patient the disease stage, based on each modality (WB-PET/CT, WB-DWI and WB-PET/MRI) according to the TNM staging (31). A combination of biopsy, surgical pathology and 24-month follow-up data were used to define the ground truth pathologic disease stage for each patient. Readers were blinded to the final clinical/pathologic stage. Studies for an individual patient were considered to be concordant if the stage derived from all three imaging modalities were in agreement, otherwise it was considered discordant. A modality stage was defined correct if in agreement with the 


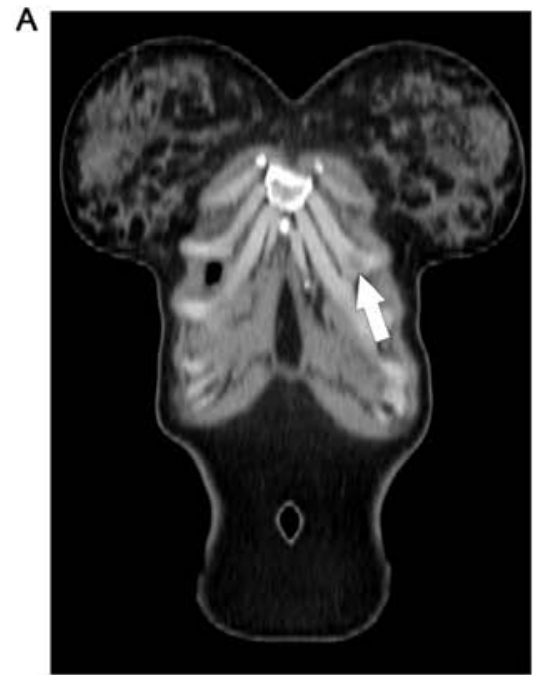

B
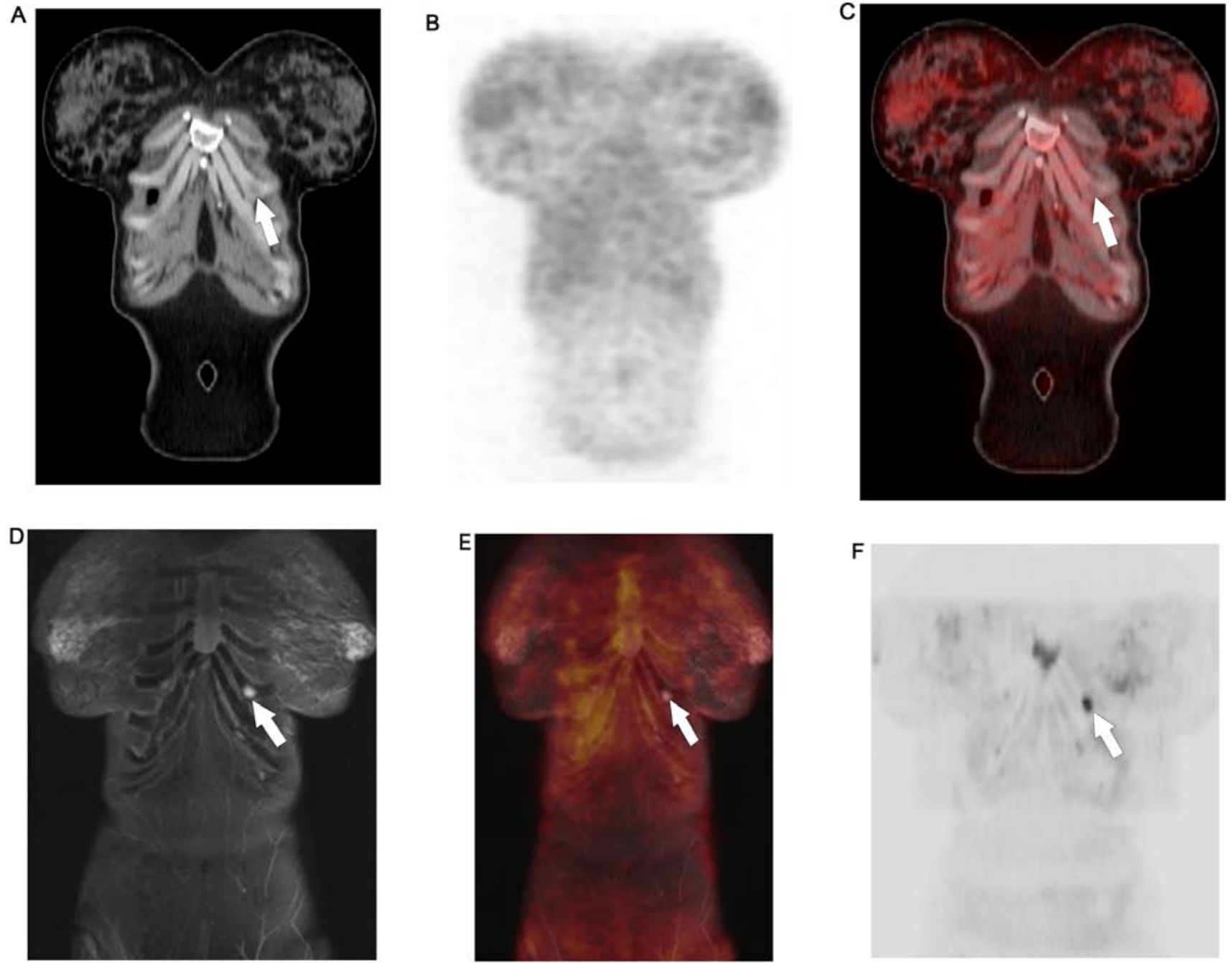

Figure 1. A 63-year-old female with stage IIB breast cancer. Coronal images from CT (A), PET (B), fused PET/CT (C), short tau inversion recovery (STIR) (D), fused PET/STIR (E), and inverted gray scale coronal DWI (F). This patient was correctly staged by PET/CT and PET/MRI, but misclassified as stage IV by DWI (arrow) due to a cartilaginous island in a left anterior rib that retained high signal intensity on the 800-b-value diffusion-weighted images. The lesion did not exhibit increased uptake on PET and did not demonstrate aggressive tumor features on CT or MR anatomic imaging.

clinical/pathological stage, otherwise it was considered incorrect.

Standard of reference. Pathology served as primary standard of reference. In the case of non-availability of pathology, imaging follow-up, lasting at least two years, served as secondary standard of reference.

Statistical analysis. The three methods (WB-PET/CT, WB-DWI and WB-PETMR) were compared pairwise using the McNemar's test, with Bonferroni adjustment for multiple comparisons.

\section{Results}

Patient demographics. A total of 191 patients with non-treated ductal invasive breast cancer underwent same-day PET/CT and PET/MRI imaging between February 2012 and December 2015. One hundred and forty patients were excluded for the following reasons: 63 for having been included in previous
PET/MRI studies with possibility of patient recall by the readers and 77 for absence of follow-up or pathology confirmation. Therefore, the final population consisted of 51 patients. The average age of study participants was 53 years with a standard deviation of 14 years (age range, 20-71 years). Final disease stage was IIA in 8 patients, IIB in 12 , IIIA in 4 , IIIC in 7 and stage IV in 20 patients.

Staging by standard of reference. Pathology served as standard of reference for 42 patients: 31 patients with stages II and III, 6 patients with oligometastatic stage IV, 5 patients with polimetastatic stage IV.

Follow-up imaging lasting $\geq 24$ months served as standard of reference for 9 patients with polimetastatic stage IV.

Final staging was stage IIA 1 patient; stage IIB 12 patients; stage IIC 7 patients; stage IIIA 4 patients; stage IIIC 7 patients; and stage IV 20 patients.

Classification concordance. Thirty-three patients (65\%) were correctly and concordantly staged by WB-PET/CT, WB-DWI 


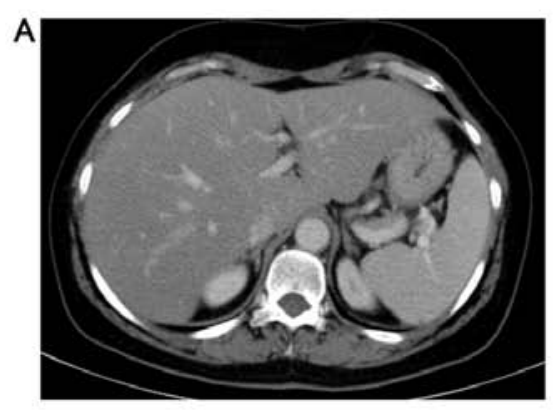

B
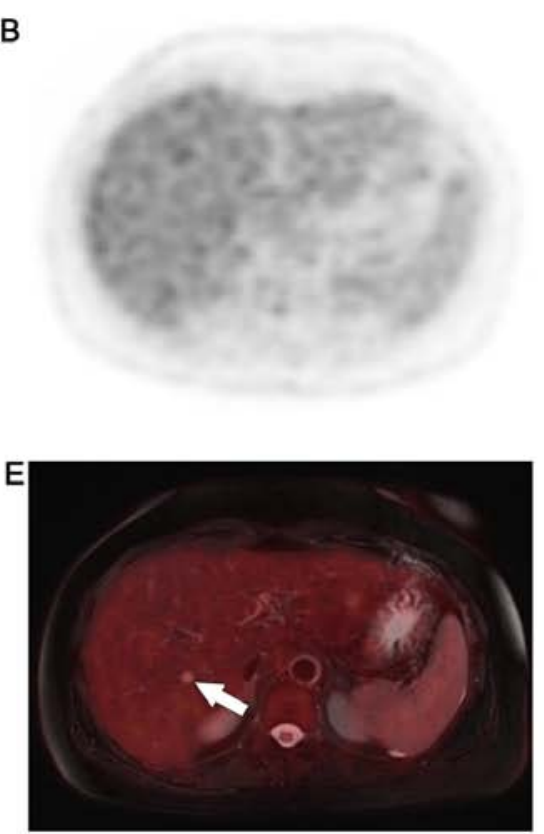
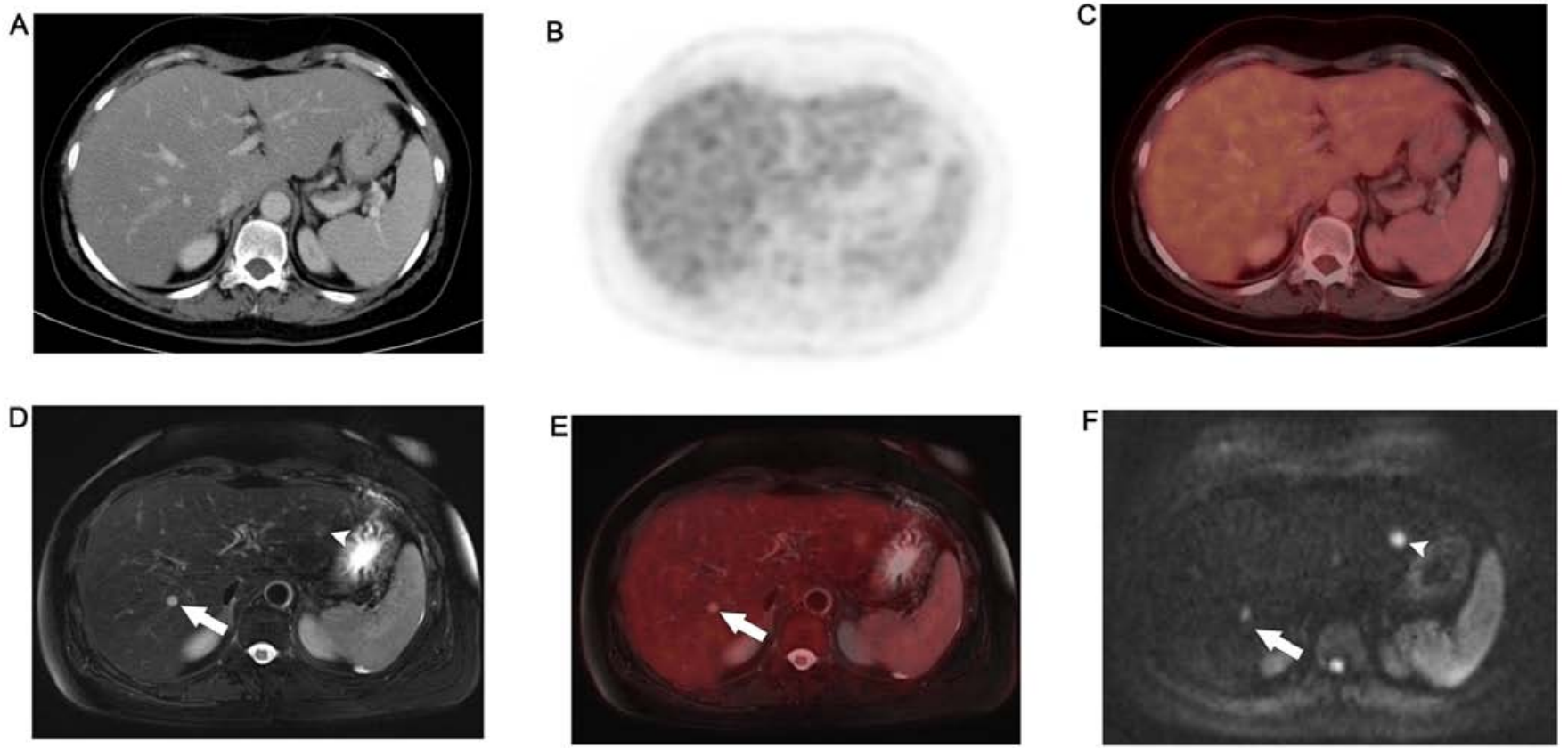

Figure 2. A 47-year-old female with stage IV breast cancer. Axial images from CT (A), PET (B), fused PET/CT (C), T2 weighted fat saturated FSE (D), fused PET/T2w fast spin echo (FSE) (E) and axial DWI (F). The metastasis in the right lobe of the liver (arrows) was not detected by PET/CT but identified on the T2 weighted fat saturated FSE acquisition and DWI. DWI disclosed also a second lesion (arrowhead) in the left lobe of the liver that was less evident in (D) and invisible on PET/CT.

Table I. Classification concordance.

\begin{tabular}{lcc}
\hline $\begin{array}{l}\text { Standard of } \\
\text { reference }\end{array}$ & $\begin{array}{c}\text { Concordant staging } \\
(34 / 51 \text { patients })\end{array}$ & $\begin{array}{c}\text { Discordant staging } \\
(17 / 51 \text { patients })\end{array}$ \\
\hline Stage IIA & - & $1(2 \%)$ \\
Stage IIB & $8(16 \%)$ & $4(8 \%)$ \\
Stage IIC & $7(14 \%)$ & - \\
Stage IIIA & - & $4(8 \%)$ \\
Stage IIIC & $4(8 \%)$ & $3(6 \%)$ \\
Stage IV & $14(27 \%)$ & $6(12 \%)$ \\
\hline
\end{tabular}

and WB-PET/MRI: 7 patients with stage IIA, 8 patients with stage IIB, 4 patients with stage IIIC and 14 patients with stage IV disease (Table I and Fig. 1).

One stage IIIA patient was incorrectly and concordantly miss-staged as IV by all modalities (Table II). Discordant staging was reported in 17 patients (33\%): 1 patient with stage IIA, 4 patients with stage IIB, 4 patients with stage IIIA, 3 patients with stage IIIC and 6 patients with stage IV disease (Tables I-II and Fig. 2).

Staging misclassification by imaging modality. To assess the performance of each imaging modality in staging newly diagnosed breast cancer, the occurrence of staging misclassification was determined for each examination: WB-DWI miss-staged 8 patients, WB-PET/CT miss-staged 13 patients and WB-PET/MRI miss-staged 1 patient.

WB-PET/MR detected FDG avid lung metastases and left liver lobe metastases that were not appreciated on WB-DWI.
Moreover, it ascertained the benign nature of lesions that, due to T2 shine-trough, retained high signal on high b-values DWI.

WB-PET/MRI identified non-FDG avid permeative bony metastases and sub-centimeter hepatic metastases that were not appreciated on WB-PET/CT. Details are provided in Table III.

Staging performance of WB-PET/CT, WB-DWI and $W B-P E T / M R I$. The staging accuracy of WB-PET/CT was $75 \%$, of WB-DWI was $84 \%$ and of WB-PET/MRI was $98 \%$ (Table III). WB-PET/MRI vs. WB-PET/CT differ significantly in their agreement with the true stage, with adjusted P-value of 0.005 . On the other hand, the differences between WB-PET/MR and WB-DWI $(\mathrm{P}=0.14)$ and between WB-PET/CT and WB-DWI $(\mathrm{P}=0.27)$ were not statistically significant.

\section{Discussion}

In the present study, we assessed the performance of whole body diffusion-weighted imaging (WB-DWI), whole body positron emission tomography with computed tomography (WB-PET/CT), and whole body positron emission tomography with magnetic resonance imaging (WB-PET/MRI) in patients with untreated invasive ductal breast cancer. All three modalities correctly staged the cancer in $64 \%$ of patients. In the patients with discordant staging among the imaging modalities, our results show superior staging accuracy of WB-PET/MRI in staging metastatic disease, when compared to the other modalities being assessed.

In particular, when compared to WB-DWI alone, PET/MR performed better both in detecting FDG avid lung metastases 


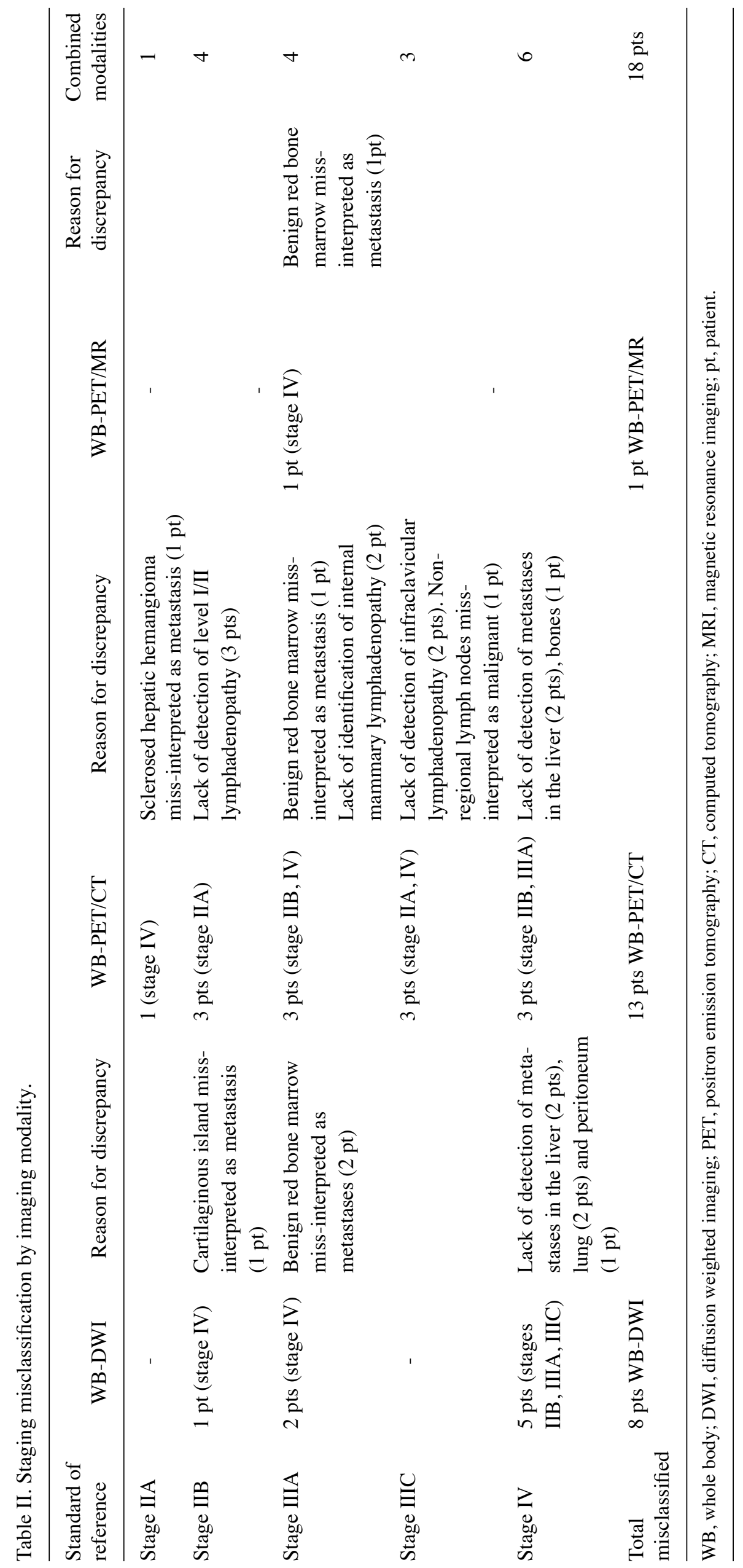


Table III. Staging performance of the assessed imaging modalities.

\begin{tabular}{lcc}
\hline & Patients correctly staged $(\mathrm{n}=51)$ & Accuracy \\
\hline WB-PET/CT & 38 & $75 \%$ \\
WB-DWI & 43 & $84 \%$ \\
WB-PET/MRI & 50 & $98 \%$ \\
\hline
\end{tabular}

WB, whole body; DWI, diffusion weighted imaging; PET, positron emission tomography; CT, computed tomography; MRI, magnetic resonance imaging.

and left liver lobe metastases, and in ruling out malignancy in the case of T2 shine-trough retention of signal in some benign lesions. This was the result of the combined information from FGD uptake and the entire setting of MR sequences of our protocol. However, these differences were not statistically significant.

Moreover, since FDG-PET is the same in both WB-PET/CT and WB-PET/MRI, the improved staging performance of WB-PET/MR is likely due to the higher sensitivity of MRI in detecting non-FDG avid lesions, as we found in the case of permeative bony and sub-centimeter hepatic metastases.

The role of PET/MRI in breast cancer staging is under investigation. A study examining the performance of PET/MRI in 36 patients with breast cancer reported correlation of standardized uptake values as measured on PET/MRI and PET/CT in primary and metastatic cancerous lesions (22). Another study in 36 patients with breast cancer showed superior performance of PET/MRI in detecting metastatic lesions, when compared to PET (23). Furthermore, PET/MRI changed management decisions in one third of the patients being studied, when compared to the initial clinical staging (23). Our results add to the existing body of literature by providing new evidence that PET/MRI outperforms PET/CT and DWI in staging patients with breast cancer.

PET/MRI has been shown to add complementary metabolic information to prostate and gynecologic MR imaging, improving the diagnosis and management of prostate and gynecologic cancer patients (32-37). Similarly, PET/MRI accurately staged 28 patients with lymphoma, when compared to PET/CT (15). Other studies have also shown superior performance of PET/MRI in diagnosing primary head and neck, bone and soft tissue lesions and for detecting metastatic disease in the brain, liver and bone $(15-18,26)$. A recent study provided evidence that PET/MRI contributes to the clinical management of cancer patients more often than PET/CT (20).

Early and appropriate staging of breast cancer is especially important in the management of patients with this disease. Available data suggest longer survival and improved quality of life with early detection of metastatic disease (38-40). Our data suggest that PET/MRI is well-positioned to aid in the staging of breast cancer patients at the time of their initial diagnosis. PET/MRI performed particularly well in accurately staging advanced disease, where a higher proportion of discordant staging was reported by other modalities in this study (stage IIIA and higher). PET/MRI might have the potential to play a critical role in affecting treatment decisions and management in this patient population.

The present study has several limitations, including the small number of patients and the potential selection bias introduced by enrolling only untreated ductal invasive breast cancers. These results might not be applicable to other breast cancer subtypes or to treated patients. A larger study would be needed to validate our findings. In this study, the availability of multiple MR sequences combined with PET helped compensate for the limitations intrinsic to stand-alone sequences. For example, DWI helped in detecting liver lesions, and PET was useful in assessing sub-centimeter metastases in the liver and in lymph nodes.

An additional limitation might have been related to the delta time with PET/CT, acquired $\sim 60$ min after FDG injection and PET/MRI, acquired $\sim 90$ min after FDG injection. Our longer incubation time for PET/MR is explained by the legal and IRB requirements that mandated us to acquire PET/MR after a standard of care PET-CT obtained at $60 \mathrm{~min}$ after FDG injection, before being allowed to acquire any PET-MR study. Although delayed PET acquisitions might demonstrate lower background activity and improved lesion visibility, there is no consensus if this translates into improved accuracy (41-43). However, it is unlikely that this might have influenced the FDG uptake obtained by PET/MR. The PET/MR reconstruction software automatically corrects for incubation time for each bed position. Moreover, several studies have demonstrated comparable performance between $\mathrm{PET} / \mathrm{CT}$ and subsequently acquired PET/MR as quantified by SUV measurements $(22,44,45)$.

Finally, in the present study, the guidelines of the European Association of Nuclear Medicine (46) were used to dictate image acquisition protocols based on local clinical standards.

In conclusion, PET/MRI outperforms PET/CT and is more accurate in staging untreated patients with invasive ductal carcinoma. PET/MRI has the potential to affect clinical decision making and management of breast cancer patients, and should be considered in the initial staging of this disease.

\section{References}

1. (NCCN) NCCN: Clinical Practice Guidelines in Oncology, 2016, accessed November 29, 2016.

2. Brennan ME and Houssami N: Evaluation of the evidence on staging imaging for detection of asymptomatic distant metastases in newly diagnosed breast cancer. Breast 21: 112-123, 2012.

3. Albano D, Patti C, La Grutta L, Agnello F, Grassedonio E, Mulè A, Cannizzaro G, Ficola U, Lagalla R, Midiri M, et al: Comparison between whole-body MRI with diffusion-weighted imaging and PET/CT in staging newly diagnosed FDG-avid lymphomas. Eur J Radiol 85: 313-318, 2016.

4. Jambor I, Kuisma A, Ramadan S, Huovinen R, Sandell M, Kajander S, Kemppainen J, Kauppila E, Auren J, Merisaari H, et al: Prospective evaluation of planar bone scintigraphy, SPECT, SPECT/CT, ${ }^{18} \mathrm{~F}-\mathrm{NaF}$ PET/CT and whole body $1.5 \mathrm{~T}$ MRI, including DWI, for the detection of bone metastases in high risk breast and prostate cancer patients: SKELETA clinical trial. Acta Oncol 55: 59-67, 2016.

5. Albano D, Patti C, Lagalla R, Midiri M and Galia M: Wholebody MRI, FDG-PET/CT, and bone marrow biopsy, for the assessment of bone marrow involvement in patients with newly diagnosed lymphoma. Journal of magnetic resonance imaging. J Magn Reson Imaging 45: 1082-1089, 2016. 
6. Littooij AS, Kwee TC, Barber I, Granata C, Vermoolen MA, Enríquez G, Zsíros J, Soh SY, de Keizer B, Beek FJ, et al: Whole-body MRI for initial staging of paediatric lymphoma: Prospective comparison to an FDG-PET/CT-based reference standard. Eur Radiol 24: 1153-1165, 2014.

7. Park SH, Moon WK, Cho N, Chang JM, Im SA, Park IA, Kang KW, Han W and Noh DY: Comparison of diffusionweighted MR imaging and FDG PET/CT to predict pathological complete response to neoadjuvant chemotherapy in patients with breast cancer. Eur Radiol 22: 18-25, 2012.

8. Heusner TA, Kuemmel S, Koeninger A, Hamami ME, Hahn S, Quinsten A, Bockisch A, Forsting M, Lauenstein T, Antoch G, et al: Diagnostic value of diffusion-weighted magnetic resonance imaging (DWI) compared to FDG PET/CT for whole-body breast cancer staging. Eur J Nucl Med Mol Imaging 37: 1077-1086, 2010.

9. Wehner J, Weissler B, Dueppenbecker PM, Gebhardt P, Goldschmidt B, Schug D, Kiessling F and Schulz V: MR-compatibility assessment of the first preclinical PET-MRI insert equipped with digital silicon photomultipliers. Phys Med Biol 60: 2231-2255, 2015

10. Delso G, Fürst S, Jakoby B, Ladebeck R, Ganter C, Nekolla SG, Schwaiger $M$ and Ziegler SI: Performance measurements of the Siemens mMR integrated whole-body PET/MR scanner. J Nucl Med 52: 1914-1922, 2011.

11. Delso G and Ziegler S: PET/MRI system design. Eur J Nucl Med Mol Imaging 36 (Suppl 1): S86-S92, 2009.

12. Yoon HS, Ko GB, Kwon SI, Lee CM, Ito M, Chan Song I, Lee DS, Hong SJ and Lee JS: Initial results of simultaneous PET/MRI experiments with an MRI-compatible silicon photomultiplier PET scanner. J Nucl Med 53: 608-614, 2012.

13. Boss A, Bisdas S, Kolb A, Hofmann M, Ernemann U, Claussen CD, Pfannenberg C, Pichler BJ, Reimold M and Stegger L: Hybrid PET/MRI of intracranial masses: Initial experiences and comparison to PET/CT. J Nucl Med 51: 1198-1205, 2010.

14. Boss A, Stegger L, Bisdas S, Kolb A, Schwenzer N, Pfister M, Claussen CD, Pichler BJ and Pfannenberg C: Feasibility of simultaneous PET/MR imaging in the head and upper neck area. Eur Radiol 21: 1439-1446, 2011.

15. Heacock L, Weissbrot J, Raad R, Campbell N, Friedman KP, Ponzo F and Chandarana H: PET/MRI for the evaluation of patients with lymphoma: Initial observations. AJR Am J Roentgenol 204: 842-848, 2015.

16. Drzezga A, Souvatzoglou M, Eiber M, Beer AJ, Fürst S, Martinez-Möller A, Nekolla SG, Ziegler S, Ganter C, Rummeny EJ, et al: First clinical experience with integrated whole-body PET/MR: Comparison to PET/CT in patients with oncologic diagnoses. J Nucl Med 53: 845-855, 2012.

17. Buchbender C, Heusner TA, Lauenstein TC, Bockisch A and Antoch G: Oncologic PET/MRI, part 2: Bone tumors, soft-tissue tumors, melanoma, and lymphoma. J Nucl Med 53: 1244-1252, 2012.

18. Buchbender C, Heusner TA, Lauenstein TC, Bockisch A and Antoch G: Oncologic PET/MRI, part 1: Tumors of the brain, head and neck, chest, abdomen, and pelvis. J Nucl Med 53: 928-938, 2012.

19. Huellner MW, Appenzeller P, Kuhn FP, Husmann L, Pietsch CM, Burger IA, Porto M, Delso G, von Schulthess GK and Veit-Haibach P: Whole-body nonenhanced PET/MR versus PET/ $\mathrm{CT}$ in the staging and restaging of cancers: Preliminary observations. Radiology 273: 859-869, 2014

20. Catalano OA, Rosen BR, Sahani DV, Hahn PF, Guimaraes AR, Vangel MG, Nicolai E, Soricelli A and Salvatore M: Clinical impact of PET/MR imaging in patients with cancer undergoing same-day PET/CT: Initial experience in 134 patients - a hypothesis-generating exploratory study. Radiology 269: 857-869, 2013

21. Lee SI, Catalano OA and Dehdashti F: Evaluation of gynecologic cancer with MR imaging, ${ }^{18} \mathrm{~F}-\mathrm{FDG}$ PET/CT, and PET/MR imaging. J Nucl Med 56: 436-443, 2015.

22. Pace L, Nicolai E, Luongo A, Aiello M, Catalano OA, Soricelli A and Salvatore M: Comparison of whole-body PET/CT and PET/MRI in breast cancer patients: Lesion detection and quantitation of ${ }^{18} \mathrm{~F}$-deoxyglucose uptake in lesions and in normal organ tissues. Eur J Radiol 83: 289-296, 2014.
23. Taneja S, Jena A, Goel R, Sarin R and Kaul S: Simultaneous whole-body ${ }^{18} \mathrm{~F}$-FDG PET-MRI in primary staging of breast cancer: A pilot study. Eur J Radiol 83: 2231-2239, 2014

24. Aklan B, Paulus DH, Wenkel E, Braun H, Navalpakkam BK, Ziegler S, Geppert C, Sigmund EE, Melsaether A and Quick HH: Toward simultaneous PET/MR breast imaging: Systematic evaluation and integration of a radiofrequency breast coil. Med Phys 40: 024301, 2013.

25. Dregely I, Lanz T, Metz S, Mueller MF, Kuschan M, Nimbalkar M, Bundschuh RA, Ziegler SI, Haase A, Nekolla SG, et al: A 16-channel MR coil for simultaneous PET/MR imaging in breast cancer. Eur Radiol 25: 1154-1161, 2015.

26. Catalano OA, Nicolai E, Rosen BR, Luongo A, Catalano M, Iannace C, Guimaraes A, Vangel MG, Mahmood U, Soricelli A, et al: Comparison of CE-FDG-PET/CT with CE-FDG-PET/MR in the evaluation of osseous metastases in breast cancer patients. Br J Cancer 112: 1452-1460, 2015.

27. Lim I, Noh WC, Park J, Park JA, Kim HA, Kim EK, Park KW, Lee SS, You EY, Kim KM, et al: The combination of FDG PET and dynamic contrast-enhanced MRI improves the prediction of disease-free survival in patients with advanced breast cancer after the first cycle of neoadjuvant chemotherapy. Eur J Nucl Med Mol Imaging 41: 1852-1860, 2014

28. Baba S, Isoda T, Maruoka Y, Kitamura Y, Sasaki M, Yoshida T and Honda H: Diagnostic and prognostic value of pretreatment SUV in ${ }^{18} \mathrm{~F}-\mathrm{FDG} / \mathrm{PET}$ in breast cancer: Comparison with apparent diffusion coefficient from diffusion-weighted MR imaging. J Nucl Med 55: 736-742, 2014.

29. Miyake KK, Nakamoto Y, Kanao S, Tanaka S, Sugie T, Mikami Y, Toi M and Togashi K: Journal Club: Diagnostic value of ${ }^{18} \mathrm{~F}-\mathrm{FDG}$ PET/CT and MRI in predicting the clinicopathologic subtypes of invasive breast cancer. AJR Am J Roentgenol 203: 272-279, 2014.

30. de Galiza Barbosa F, Delso G, Zeimpekis KG, Ter Voert E, Hüllner M, Stolzmann P and Veit-Haibach P: Evaluation and clinical quantification of neoplastic lesions and physiological structures in TOF-PET/MRI and non-TOF/MRI - a pilot study. Q J Nucl Med Mol Imaging: May 12, 2015 (Epub ahead of print).

31. Sobin LH, Gospodarowicz MK and Wittekind CH: TNM Classification of Malignant Tumours. 7th edition. WileyBlackwell, Chichester, 2010.

32. Wetter A, Lipponer C, Nensa F, Beiderwellen K, Olbricht T, Rübben H, Bockisch A, Schlosser T, Heusner TA and Lauenstein TC: Simultaneous ${ }^{18} \mathrm{~F}$ choline positron emission tomography/magnetic resonance imaging of the prostate: Initial results. Invest Radiol 48: 256-262, 2013.

33. Wetter A, Nensa F, Schenck M, Heusch P, Pöppel T, Bockisch A, Forsting M, Schlosser TW, Lauenstein TC and Nagarajah J: Combined PET imaging and diffusion-weighted imaging of intermediate and high-risk primary prostate carcinomas with simultaneous $\left[{ }^{18} \mathrm{~F}\right]$ choline PET/MRI. PLoS One 9: e101571, 2014.

34. de Perrot T, Rager O, Scheffler M,Lord M,Pusztaszeri M, Iselin C, Ratib $\mathrm{O}$ and Vallee JP: Potential of hybrid ${ }^{18} \mathrm{~F}$-fluorocholine PET/MRI for prostate cancer imaging. Eur J Nucl Med Mol Imaging 41: 1744-1755, 2014.

35. Grueneisen J, Beiderwellen K, Heusch P, Gratz M, SchulzeHagen A, Heubner M, Kinner S, Forsting M, Lauenstein T, Ruhlmann V, et al: Simultaneous positron emission tomography/ magnetic resonance imaging for whole-body staging in patients with recurrent gynecological malignancies of the pelvis: A comparison to whole-body magnetic resonance imaging alone. Invest Radiol 49: 808-815, 2014.

36. Grueneisen J, Schaarschmidt BM, Beiderwellen K, SchulzeHagen A, Heubner M, Kinner S, Forsting M, Lauenstein T, Ruhlmann V and Umutlu L: Diagnostic value of diffusionweighted imaging in simultaneous ${ }^{18} \mathrm{~F}-\mathrm{FDG}$ PET/MR imaging for whole-body staging of women with pelvic malignancies. J Nucl Med 55: 1930-1935, 2014.

37. Souvatzoglou M, Eiber M, Takei T, Fürst S, Maurer T, Gaertner F, Geinitz H, Drzezga A, Ziegler S, Nekolla SG, et al: Comparison of integrated whole-body $\left[{ }^{11} \mathrm{C}\right]$ choline PET/MR with PET/CT in patients with prostate cancer. Eur J Nucl Med Mol Imaging 40: 1486-1499, 2013

38. Selzner M, Morse MA, Vredenburgh JJ, Meyers WC and Clavien PA: Liver metastases from breast cancer: Long-term survival after curative resection. Surgery 127: 383-389, 2000. 
39. Vogl TJ, Naguib NNN, Nour-Eldin N-EA, Mack MG, Zangos S, Abskharon JE and Jost A: Repeated chemoembolization followed by laser-induced thermotherapy for liver metastasis of breast cancer. AJR Am J Roentgenol 196: W66-W72, 2011.

40. Alexander E III, Moriarty TM, Davis RB, Wen PY, Fine HA, Black PM, Kooy HM and Loeffler JS: Stereotactic radiosurgery for the definitive, noninvasive treatment of brain metastases. J Natl Cancer Inst 87: 34-40, 1995.

41. Chen YM, Huang G, Sun XG, Liu JJ, Chen T, Shi YP and Wan LR: Optimizing delayed scan time for FDG PET: Comparison of the early and late delayed scan. Nucl Med Commun 29: 425-430, 2008.

42. Cheng G, Torigian DA, Zhuang $\mathrm{H}$ and Alavi A: When should we recommend use of dual time-point and delayed time-point imaging techniques in FDG PET? Eur J Nucl Med Mol Imaging 40: 779-787, 2013

43. Laffon E, de Clermont $H$, Begueret $H$, Vernejoux JM, Thumerel M, Marthan R and Ducassou D: Assessment of dualtime-point ${ }^{18} \mathrm{~F}$-FDG-PET imaging for pulmonary lesions. Nucl Med Commun 30: 455-461, 2009.
44. Atkinson W, Catana C, Abramson JS, Arabasz G, McDermott S, Catalano O, Muse V, Blake MA, Barnes J, Shelly M, et al: Hybrid FDG-PET/MR compared to FDG-PET/CT in adult lymphoma patients. Abdom Radiol (NY) 41: 1338-1348, 2016.

45. Pujara AC, Raad RA, Ponzo F, Wassong C, Babb JS, Moy L and Melsaether AN: Standardized uptake values from PET/MRI in metastatic breast cancer: An organ-based comparison with PET/ CT. Breast J 22: 264-273, 2016.

46. Boellaard R, O'Doherty MJ, Weber WA, Mottaghy FM, Lonsdale MN, Stroobants SG, Oyen WJ, Kotzerke J, Hoekstra OS, Pruim J, et al: FDG PET and PET/CT: EANM procedure guidelines for tumour PET imaging: version 1.0. Eur J Nucl Med Mol Imaging 37: 181-200, 2010. 